\title{
Surgical Consent during COVID Pandemic: COVID Times-Surgical Consent Checklist
}

\author{
Toney Jose ${ }^{1}$ (1) $\cdot$ Arya Joy $^{2}$ \\ Received: 22 June 2020 / Accepted: 29 July 2020 / Published online: 3 August 2020 \\ (C) Association of Surgeons of India 2020
}

\begin{abstract}
COVID-19 caused many countries to stop their elective procedures to allow preservation of resources for COVID-19 care. With restriction being gradually lifted, the surgical services have to face the pending burden of elective cases alongside the pandemic. The true impact of the pandemic and the COVID-19 on perioperative outcomes is still being discovered. This demands a COVID-specific consenting process in addition to the routine surgical consent, to ensure that the patients are able to make informed decisions. The first ever COVID-specific checklist for surgical consent 'COVID times - surgical consent checklist' is introduced. This checklist enables the surgeon to ensure that a discussion detailing the impact of COVID-19 on surgical services is made. It also acts as a documentation of the discussions carried out during the consenting process.
\end{abstract}

Keywords COVID consent checklist $\cdot$ Surgical consent $\cdot$ Consent $\cdot$ Checklist

\section{Introduction}

Since the detection of COVID-19 in Wuhan in December 2019, the virus has spread over majority of the countries. While the virus continues to spread, the surgical diseases continue to occur. Initially many countries opted to postpone elective noncancer cases, to conserve healthcare workers and hospital resources, and to prepare the infrastructure to face the pandemic. However, as lockdown restrictions are being released, the healthcare set up is faced with the pent-up burden of postponed surgical procedures among continuing surges in COVID-19 cases. An essential aspect of restarting the surgical services is the consenting process. The 'COVID times-surgical consent checklist' that is introduced here enables the surgeon to have detailed discussions with the patient, covering all aspects of surgical care during the pandemic.

Toney Jose

toneyonly@gmail.com

1 Department of Surgical Gastroenterology, Bangalore Medical College and Research Institute, Bangalore, India

2 Department of General Surgery, Government Medical College, Kottayam, Kerala, India

\section{Surgical Consent in COVID}

Informed consent is an integral part of surgical procedure, where the surgeon weaves data extracted from literature to inform the patient about the available options and help the patient to take an informed decision on the treatment direction. COVID-19 provides a unique scenario, where the understanding of effects of undetected and detected COVID-19 on the perioperative outcomes - in association with surgical and anaesthetic stress - is limited. Similarly, the possibility of nosocomial transmission while in hospital environment is unknown.

Literature on surgical outcomes of perioperative COVID-19 is gradually accumulating. The first report from Wuhan, which reported the outcomes of 34 patients who underwent elective surgeries during the incubation period of SARS-CoV-2, noted a $100 \%$ incidence of postoperative pneumonia-with 11 (32\%) developing ARDS [1]. There was an overall mortality rate of $21 \%$ (7 of 34 ). All mortalities were due to respiratory failure. The mortality rate in patients who underwent elective surgery was higher that the reported overall case fatality of $2.3 \%$ in COVID-19 patients [2].

CovidSurg collaborative in its report described the outcomes of 1138 patients with perioperative COVD-19 infection from 24 countries. The overall 30-day mortality among patients was $24.8 \%$, with $19.1 \%$ in elective surgeries and 
$26 \%$ in emergency surgeries. There was an overall incidence of $51.2 \%$ for pulmonary complications. Increased age, male sex, higher ASA grade, presence of one or more comorbidities, emergency surgery, cancer surgery, and major surgery were seen as predictors of 30 -day mortality. The diagnosis of SARS-CoV-2 in preoperative vs the postoperative period did not show any difference in outcome [3].

These perioperative outcomes are in contrast to previously reported overall mortality of $9.6 \%$ in the National Emergency Laparotomy Audit [4]. Even the high-risk group in NELA had only $16.9 \% 30$-day mortality. The GlobalSurg collaborative noted that emergency abdominal surgery had a 30-day mortality of $4.5 \%$ in high-income countries, $6.0 \%$ in middleincome countries, and $8.7 \%$ in low-income countries [5]. And in the POPULAR study, the rate of pulmonary complications was only $7.6 \%$ [6].

Available evidence suggests that the tests currently available have false negative rates which vary depending on the time since infection. On the day of symptom onset, the median false negative rate was seen to be $38 \%$. This decreased to $20 \%$ by day 8 and then again increased [7]. Thus, the possibility of a false negative asymptomatic patient being subjected to an elective surgical procedure does exist.

\section{COVID Times-Surgical Consent Checklist}

These issues highlight the need for effective discussions between the surgeon and the patient, on how COVID-19 affects the surgical services and perioperative outcomes. Additional consent for impact of COVID-19 on outcomes has been recommended by various associations [8]. Few COVID-19-specific consent forms have been published online for use [9]. However, it is to be noted that the consent forms should be regional and specific to each institute, detailing the various aspects of patient care during the pandemic. To help the surgeon carryout effective discussions with the patient about all aspects of COVID-19 in surgical scenario, we have described the first ever 'COVID times - surgical consent checklist' (Fig. 1). Use of this checklist during the discussion will allow surgeons to cover all pertinent aspects of consenting during the pandemic. 'COVID times - surgical con-

\section{COVID TIMES - SURGICAL CONSENT CHECKLIST}

\begin{abstract}
Information on coronavirus and its infectivity
Disease course and general death rate in the country

Need for preoperative testing for SARS-COV2

Availability of testing facility for SARS-COV2 in the hospital

False negative rate in RT-PCR or test used to diagnose SARS-COV2

Changes in sensitivity depending on time of testing since infection

Possible risk of nosocomial infection with SARS-COV2 because social distancing from caregivers is inherently imperfect despite best efforts and meticulous attention to infection control

Possible risk of cross infection of SARS-COV2 from other patients

Increased complications, ICU care and mortality rates with perioperative infection

Risks vs benefits in delaying otherwise medically indicated procedures

Available alternative (non surgical) options for treatment
\end{abstract}

Signature
Name
Mobile No
Aadhar No
Date
Place

Fig. 1 COVID times - surgical consent checklist: Includes all areas of discussion between the surgeon and the patient that enables the patient to undertake an informed decision regarding surgical procedure during
Potential shortages in staff and logistics - impact on perioperative care and experience

Measures taken by hospital to decrease risk of nosocomial infection

Increased cost of hospital services due to extra measures

Restrictions placed on patients and family -visitor restriction policies

Patient and attendants were carefully explained the do's and dont's to prevent any cross infection of coronavirus during the course of surgical treatment

Patients are allowed to make advance healthcare directives regarding the treatment of postoperative complications

Discussions held were carefully documented

Patients and attendants understands that this is an area of ongoing uncertainty and investigation

A document with list of instructions, do's and dont's and all that is written above was handed over to the patient and his/her caregivers

Witness $\quad$ Surgeon

COVID pandemic. The checklist helps surgeons to avoid missing out on important aspects of consenting. It also acts as a documentation of the discussions done during consenting process 
sent checklist' can also be used as a documentation of the consent process and discussions made.

\section{Challenges in Consent Process}

Non-verbal expression is an integral part of effective communication and consenting process. Challenges to effective communication are presented by the use of personal protective equipment (PPE) which limits non-verbal expression. This can cause additional distress to patients, as they are not familiar with such looks of their doctors. Also, patient counselling carries a risk of infection because maintaining 2-m distance between the surgeon and the patient may not be easy to follow. Finally, the limited data available might not be enough to make informed decisions.

\section{Conclusion}

COVID-19 has drastically affected the patient care with regard to elective surgical services. As institutions attempt to restart their surgical services, a balance has to be weighed with regard to perioperative outcomes in setting of undetected COVID-19. The decision process involves active participation of the surgeon and the patient especially with regard to implications of the pandemic on the perioperative outcomes and the hospital logistics. The COVID times - surgical consent checklist will help guide the decision process by ensuring that all aspects are discussed. Furthermore, it can also act as a documentation of the discussion process.

Authors' Contributions Both authors contributed to the study conception and design. Material preparation and data collection were performed by both authors. The first draft of the manuscript was written by Toney Jose. Both authors read and approved the final manuscript.

Data Availability Not applicable.

\section{Compliance with Ethical Standards}

Conflict of Interest The authors declare that they have no conflict of interest.
Ethics Approval Not applicable.

Consent to Participate Not applicable.

Consent for Publication Not applicable.

Code Availability Not applicable.

\section{References}

1. Lei S, Jiang F, Su W et al (2020) Clinical characteristics and outcomes of patients undergoing surgeries during the incubation period of COVID-19 infection. EClinicalMedicine 100331. https://doi.org/ 10.1016/j.eclinm.2020.100331

2. Wu Z, McGoogan JM (2020) Characteristics of and important lessons from the coronavirus disease 2019 (COVID-19) outbreak in China: summary of a report of 72314 cases from the Chinese Center for Disease Control and Prevention. JAMA. https://doi.org/ 10.1001/jama.2020.2648

3. COVIDSurg Collaborative (2020) Mortality and pulmonary complications in patients undergoing surgery with perioperative SARSCoV-2 infection: an international cohort study. Lancet. https://doi. org/10.1016/S0140-6736(20)31182-X

4. NELA Project Team (2019) Fifth patient report of the National Emergency Laparotomy Audit. https://www.nela.org.uk/ downloads/EMBARGO - The Fifth Patient Report of the NELA 2019 - 27-11-19.pdf Accessed 08 June 2020

5. GlobalSurg Collaborative (2016) Mortality of emergency abdominal surgery in high-, middle- and low-income countries. Br J Surg 103: 971-988. https://doi.org/10.1002/bjs.10151

6. Kirmeier E, Eriksson LI, Lewald H et al (2019) Post-anaesthesia pulmonary complications after use of muscle relaxants (POPULAR): a multicentre, prospective observational study. Lancet Respir Med 7:129-140. https://doi.org/10.1016/S22132600(18)30294-7

7. Kucirka LM, Lauer SA, Laeyendecker O et al (2020) Variation in false-negative rate of reverse transcriptase polymerase chain reaction-based SARS-CoV-2 tests by time since exposure. Ann Intern Med. https://doi.org/10.7326/M20-1495

8. Somashekhar SP, Shivaram HV, Abhaham SJ et al (2020) ASI's consensus guidelines: $\mathrm{ABCs}$ of what to do and what not during the COVID-19 pandemic. Indian J Surg:1-11. https://doi.org/10.1007/ s12262-020-02452-z

9. Bhattacharya N, Bhattacharya K (2020) Informed consent for surgery during COVID-19. Indian J Surg 1-3. https://doi.org/10.1007/ s12262-020-02283-y

Publisher's Note Springer Nature remains neutral with regard to jurisdictional claims in published maps and institutional affiliations. 